\title{
The non-perturbative regime of cosmic structure formation
}

\author{
T. Buchert
}

\begin{abstract}
Arnold Sommerfeld Center for Theoretical Physics, Ludwig-Maximilians-Universität, Theresienstr. 37, 80333 München, Germany e-mail: buchert@theorie.physik.uni-muenchen.de
\end{abstract}

Received 23 January 2006 / Accepted 14 March 2006

ABSTRACT

\begin{abstract}
This paper focusses on the barely understood gap between the weakly nonlinear regime of structure formation and the onset of the virialized regime. While the former is accessed through perturbative calculations and the latter through virialization conditions incorporating dynamical stresses that arise in collisionless self-gravitating systems due to velocity dispersion forces, the addressed regime can only be understood through non-perturbative models. We here present an exact Lagrangian integral that provides a tool to access this regime. We derive a transport equation for the peculiar-gravitational field strength and integrate it along comoving trajectories of fluid elements. The so-obtained integral provides an exact expression that solves the longitudinal gravitational field equation in general. We argue that this integral provides a powerful approximation beyond the Lagrangian perturbative regime, and discuss its relation to known approximations, among them Lagrangian perturbation solutions including the Zel'dovich approximation and approximations for adhesive gravitational clustering, including the adhesion approximation. Furthermore, we propose an iteration scheme for a systematic analytical and numerical construction of trajectory fields. The integral may also be employed to improve inverse reconstruction techniques.
\end{abstract}

Key words. gravitation - methods: analytical - cosmology: theory - cosmology: large-scale structure of Universe

\section{Introduction}

Both the analytical understanding and the numerical simulation of the formation of cosmic structure in the Universe have substantially advanced in recent years. Early results in the development of both approaches and their comparison has improved our understanding of the building blocks of large-scale structure. Both focussed on modelling dark matter in terms of a Newtonian dust continuum, but recent developments are heading in somewhat different directions. Numerical attempts are directed towards improving spatial resolution, understanding the force distribution on a lattice, and incorporating new physics for the modelling of structure formation on galaxy halo scales (e.g., Bagla \& Padmanabhan 1997; Gabrielli et al. 2006; Bertschinger 1998; Shirokov \& Bertschinger 2006) and for the modelling of hydrodynamical effects (Steinmetz 2003). Analytical improvements have still followed the route of understanding structure formation in a self-gravitating dust continuum, but also attempt to access small-scale structure by incorporating additional forces in the Euler equation that arise from kinetic theory (e.g., Ma \& Bertschinger 2004; Buchert \& Domínguez 2005). In this work we discuss a regime that is difficult to understand and to model using both numerical and analytical strategies. The analytical insight will also shed light on and provide tools for numerical and semi-analytical techniques.

In Sect. 2 we recall the basic equations, as well as cosmological models that have been obtained previously and that help to locate the non-perturbative regime from the analytical point of view. In Sect. 3 we then formulate the key equation of the present work and integrate this equation exactly along the flow lines of continuum elements. The implications of this result are investigated in Sect. 4, and in Sect. 5 we give a discussion summary.

In this work we do not provide a comprehensive reference list, because the reader may find systematic derivations of the equations of Sect. 2 as well as a substantial list of references in a recent paper on the current status of analytical models (Buchert \& Domínguez 2005). We first work in Eulerian coordinates that are comoving with a given reference Hubble flow, i.e. a homogeneous-isotropic solution of the Euler-Newton system of equations, as is widely used in cosmology. We denote them by $\boldsymbol{q}=\boldsymbol{x} / a(t)$, where $\boldsymbol{x}$ are the Eulerian coordinates, $a(t)$ a solution of Friedmann's differential equation, and $H:=\dot{a} / a$ is Hubble's function. Later, we move to the Lagrangian picture of fluid motion and represent the comoving trajectory field of continuum elements by $\boldsymbol{q}=\boldsymbol{F}(\boldsymbol{X}, t)$, where $\boldsymbol{X}$ are the Lagrangian coordinates, which index fluid elements. The velocity field is split into a Hubble-velocity and a peculiar-velocity, $\boldsymbol{v}=\boldsymbol{v}_{\mathrm{H}}+\boldsymbol{u}$, the acceleration field into a Hubble-acceleration and a peculiaracceleration $\boldsymbol{g}=\boldsymbol{g}_{\mathrm{H}}+\boldsymbol{w}$, the density field into a background density and a density contrast $\varrho=\varrho_{\mathrm{H}}(1+\delta)$. The total (Lagrangian) time-derivative is denoted by $\frac{\mathrm{d}}{\mathrm{d} t}=\left.\partial_{\mathrm{t}}\right|_{\boldsymbol{x}}+\boldsymbol{v} \cdot \nabla_{\boldsymbol{x}}=\left.\partial_{\mathrm{t}}\right|_{\boldsymbol{q}}+\boldsymbol{u} / a \cdot \nabla_{\boldsymbol{q}}$, and sometimes by an overdot. The nabla-operator with respect to Lagrangian coordinates is denoted by $\nabla_{0}$ and sometimes by $\nabla_{\boldsymbol{X}}$.

\section{The non-perturbative regime}

In this section we recall the basic equations in a form that is helpful for our purpose and then review the known cosmological model equations within the presented framework and discuss their limits with regard to non-perturbative effects in the nonlinear regime.

\subsection{Equations for the non-perturbative regime}

For the basic system of equations, derived from coarse-graining the Newtonian kinetic equations for a system of $\mathrm{N}$ selfgravitating particles, we refer the reader to our recent paper 
(Buchert \& Domínguez 2005). We work below with hydrodynamical equations that can also be found in many cosmology textbooks and review papers (e.g., Peebles 1980; Binney \& Tremaine 1987; Sahni \& Coles 1995; Ehlers \& Buchert 1997; Bernardeau et al. 2002).

We start with recalling equations that describe the evolution of the density $\varrho$, the peculiar-velocity $\boldsymbol{u}$, and the peculiaracceleration or the peculiar-gravitational field strength $w$, obtained by coarse-graining the kinetic equations of $\mathrm{N}$ selfgravitating particles in real space and velocity space and then forming velocity moments to obtain a hydrodynamical description in Eulerian space. The zeroth velocity moment is the continuity equation,

$\frac{\mathrm{d}}{\mathrm{d} t} \varrho+3 H \varrho+\frac{1}{a} \nabla_{\boldsymbol{q}} \cdot \boldsymbol{u}=0$.

The first velocity moment provides the evolution equation for the peculiar-velocity:

$\frac{\mathrm{d}}{\mathrm{d} t} \boldsymbol{u}+H \boldsymbol{u}=\boldsymbol{w}+\frac{1}{\varrho}\left(\boldsymbol{F}-\frac{1}{a} \nabla_{\boldsymbol{q}} \cdot \boldsymbol{\Pi}\right)$.

In the above equation, the peculiar-gravitational field strength $w$ is constrained by the Newtonian field equations:

$\nabla_{\boldsymbol{q}} \times \boldsymbol{w}=\mathbf{0} ; \quad \nabla_{\boldsymbol{q}} \cdot \boldsymbol{w}=-4 \pi G \varrho_{\mathrm{H}} a \delta$.

The force $\boldsymbol{F}$ represents deviations from mean field gravity (modelled by Eqs. (1c)), and the symmetric tensor field $\Pi$ represents forces due to velocity dispersion. For $\boldsymbol{F}=\mathbf{0}$, Eq. (1b) is known as the Euler-Jeans equation in stellar system theory. The above set of equations truncates the velocity moment hierarchy, so we need models for the fields $\boldsymbol{F}$ and $\boldsymbol{\Pi}$ (e.g., as functionals of the other fields) to close the system of equations. The simplest truncation is to neglect deviations from mean field gravity and velocity dispersion altogether and study the evolution of a dust continuum. More general models for adhesive gravitational clustering including those forces are investigated in (Buchert \& Domínguez 2005).

In this work we focus on the (in the above framework) general evolution equation for the peculiar-gravitational field strength (Buchert 1989)

$$
\begin{aligned}
& \frac{\mathrm{d}}{\mathrm{d} t} \boldsymbol{w}+2 H \boldsymbol{w}-4 \pi G \varrho_{\mathrm{H}} \boldsymbol{u}=\mathcal{R} ; \\
& \mathcal{R}:=\frac{1}{a}\left[\left(\boldsymbol{u} \cdot \nabla_{\boldsymbol{q}}\right) \boldsymbol{w}-\boldsymbol{u}\left(\nabla_{\boldsymbol{q}} \cdot \boldsymbol{w}\right)+\nabla_{\boldsymbol{q}} \times \boldsymbol{T}\right],
\end{aligned}
$$

which can be obtained from Eq. (1a) by inserting the field equations (1c) and formally integrating the divergence. The resulting vector field of integration $\boldsymbol{T}$ can be determined as follows. Acting with $\nabla_{q} \times$ on Eq. (2) and subjecting $\boldsymbol{T}$ to the Coulomb gauge condition, $\nabla_{\boldsymbol{q}} \cdot \boldsymbol{T}=0$, one finds:

$$
\begin{aligned}
\Delta_{\boldsymbol{q}} \boldsymbol{T} & =4 \pi G a \nabla_{\boldsymbol{q}} \times(\varrho \boldsymbol{u})=4 \pi G a\left[\varrho \nabla_{\boldsymbol{q}} \times \boldsymbol{u}+\nabla_{\boldsymbol{q}} \varrho \times \boldsymbol{u}\right] \\
& =\left(4 \pi G a \varrho_{\mathrm{H}}-\nabla_{\boldsymbol{q}} \cdot \boldsymbol{w}\right) \nabla_{\boldsymbol{q}} \times \boldsymbol{u}+\boldsymbol{u} \times \Delta_{\boldsymbol{q}} \boldsymbol{w} .
\end{aligned}
$$

That is, $\boldsymbol{T}$ is the vector potential (up to an unimportant factor) of the peculiar-current density $\boldsymbol{j}:=\varrho \boldsymbol{u}$ :

$4 \pi G a j=-\nabla_{\boldsymbol{q}} \times \boldsymbol{T}-\left.\nabla_{\boldsymbol{q}} \frac{1}{a} \partial_{\mathrm{t}}\right|_{\boldsymbol{q}}[a \phi]$,

with $\phi$ denoting the scalar peculiar-gravitational potential. (Note that Eq. (4) is equivalent to Eq. (2) by employing the definitions $\frac{\mathrm{d}}{\mathrm{d} t} \boldsymbol{w}=\left.\partial_{t}\right|_{\boldsymbol{q}} \boldsymbol{w}+\boldsymbol{u} / a \cdot \nabla_{\boldsymbol{q}} \boldsymbol{w}$ and $\boldsymbol{w}=:-\frac{1}{a} \nabla_{\boldsymbol{q}} \phi$.) If we require $\boldsymbol{T}$ to vanish, then Equation (3) shows that, for irrotational flows, the mean flow follows the gradient of the density field ${ }^{3}$ ).

We thus have obtained a new set of equations that is equivalent to the set $(1 \mathrm{a}, 1 \mathrm{c})$, and we can write it in a form that reminds us of Maxwell's equations:

$$
\begin{array}{ll}
\frac{1}{a} \nabla_{\boldsymbol{q}} \times \boldsymbol{w}=\mathbf{0} & \frac{1}{a} \nabla_{\boldsymbol{q}} \cdot \boldsymbol{w}=-4 \\
\frac{1}{a} \nabla_{\boldsymbol{q}} \times \boldsymbol{T}=\left.\partial_{\mathrm{t}}\right|_{\boldsymbol{q}} \boldsymbol{w}-4 \pi G \boldsymbol{j} & \frac{1}{a} \nabla_{\boldsymbol{q}} \cdot \boldsymbol{T}=0 .
\end{array}
$$

For the purpose of constructing models for cosmic structure formation, one usually employs the Eqs. (1a)-(1c) and approximates the fields $\boldsymbol{F}$ and $\boldsymbol{\Pi}$. A commonly used approximation is to set $\boldsymbol{F}$ equal to zero and "masking" $\boldsymbol{\Pi}$ by its isotropic contribution, $\Pi_{i j}=: p \delta_{i j}$, together with a dynamical equation of state $p=\beta(\varrho)$ (Buchert \& Domínguez 1998). For such a closure approximation, we obtain the starting equations for a large set of approximations that have been discussed in the cosmological literature.

To recall these approximations, let us now move to the Lagrangian picture of fluid mechanics, where the Eulerian positions are viewed as a field variable depending on the initial position vectors $\boldsymbol{X}$ (the Lagrangian coordinates) and the time $t$, $\boldsymbol{q}=\boldsymbol{F}(\boldsymbol{X}, t)$. The trajectory field $\boldsymbol{F}$ represents the integral curves of the scaled peculiar-velocity field, $\boldsymbol{u}=a \dot{\boldsymbol{F}}$. We derive equations for the comoving displacement field $\boldsymbol{P}:=\boldsymbol{F}-\boldsymbol{X}$. First, we formally integrate Eq. (2) with respect to the time and obtain (Buchert \& Domínguez 2005):

$\boldsymbol{w}=\frac{\boldsymbol{W}}{a^{2}}+4 \pi G \varrho_{\mathrm{H}} a \boldsymbol{P}(\boldsymbol{X}, t)+\frac{1}{a^{2}} \int_{t_{0}}^{t} \mathrm{~d} t^{\prime} a^{2}\left(t^{\prime}\right) \mathcal{R}\left(\boldsymbol{X}, t^{\prime}\right)$,

with

$$
\boldsymbol{w}\left(\boldsymbol{X}, t_{0}\right)=: \boldsymbol{W}(\boldsymbol{X}), \quad \boldsymbol{P}\left(\boldsymbol{X}, t_{0}\right):=\mathbf{0} .
$$

The integration constant has been chosen such that the Lagrangian coordinates coincide with the comoving Eulerian coordinates at initial time ${ }^{4}$. Notice that Eq. (6a) is general and does not depend on the specific models for the forces in Eq. (1b). Inserting this general expression for $w$ into Eq. (1b) and using $\boldsymbol{u}=a \dot{\boldsymbol{P}}$ yields an evolution equation for the displacement field $\boldsymbol{P}$, which we simplify - for the sake of a more transparent discussion - to the case $\boldsymbol{F}=\mathbf{0}$ and an isotropic velocity dispersion tensor (representing the dynamical pressure by an equation of state of the form $p=\beta(\varrho)$ ); this restriction implies:

$-\frac{1}{a^{2} \varrho} \nabla_{\boldsymbol{q}} \cdot \boldsymbol{\Pi}=\frac{L_{\mathrm{J}}^{2}(\varrho)}{a^{3}} \Delta_{\boldsymbol{q}} \boldsymbol{w}$.

Above, we have introduced a density dependent Jeans' length as the product of the speed of sound $c_{\mathrm{s}}$ and the local free-fall time $t_{\mathrm{F}}:$

$L_{\mathrm{J}}:=\sqrt{\frac{\beta^{\prime}(\varrho)}{4 \pi G \varrho}}=c_{\mathrm{s}} t_{\mathrm{F}}, c_{\mathrm{s}}^{2}:=\frac{\mathrm{d} p}{\mathrm{~d} \varrho}=\beta^{\prime}(\varrho), t_{\mathrm{F}}:=\frac{1}{\sqrt{4 \pi G \varrho}}$.

${ }^{3}$ If such conditions are imposed on the problem, $\boldsymbol{T}$ becomes a harmonic vector function that can be set to zero for periodic boundary conditions, since harmonic functions are then spatially constant and can be set to zero due to the invariance of the basic equations with respect to spatially constant translations.

4 Alternatively, we could define initial displacements through $4 \pi G \varrho_{\mathrm{H}}\left(t_{0}\right) \boldsymbol{P}\left(\boldsymbol{X}, t_{0}\right)=\boldsymbol{w}\left(\boldsymbol{X}, t_{0}\right)$. This means either that we have assumed quasi-homogeneity, $\varrho\left(\boldsymbol{X}, t_{0}\right) \approx \varrho_{\mathrm{H}}\left(t_{0}\right)$ or, else, we have adopted a particular choice of Lagrangian coordinates. With this assumption we get rid of physically unimportant constant terms, which is sometimes useful, but will not be used here to avoid confusion with the exact property of the expressions derived in this paper. (For details on the possibility and advantage of this choice, see Adler \& Buchert 1999, Appendix A.) 
For this case we obtain the following evolution equation:

$$
\begin{aligned}
\ddot{\boldsymbol{P}}+ & 2 H \dot{\boldsymbol{P}}-4 \pi G \varrho_{\mathrm{H}} \boldsymbol{P}= \\
& \frac{L_{\mathrm{J}}^{2}(\varrho)}{a^{3}} \Delta_{q} w+\frac{1}{a^{2}} \int_{t_{0}}^{t} \mathrm{~d} t^{\prime} a^{2}\left(t^{\prime}\right) \mathcal{R}\left(\boldsymbol{X}, t^{\prime}\right) .
\end{aligned}
$$

\subsection{A list of known cosmological model equations}

From Eq. (9) we are able to infer a list of approximation assumptions that have been used for the construction of cosmological evolution models:

1 Setting $L_{\mathrm{J}}=0$ formally we obtain the dust model without any restriction.

2 Neglecting the residual vector field $\mathcal{R}=\mathbf{0}$, we obtain for $L_{\mathrm{J}}=0$ the equation for the longitudinal part of Lagrangian first-order perturbations (the transversal part is hidden in $\mathcal{R}=$ 0). The peculiar-gravitational field strength is then, in view of Eq. (6a), approximated through the relation:

$\boldsymbol{w}=\frac{\boldsymbol{W}}{a^{2}}+4 \pi G \varrho_{\mathrm{H}} a \boldsymbol{P}$.

3 Also neglecting the residual vector field $\mathcal{R}=\mathbf{0}$, we obtain for $L_{\mathrm{J}} \neq 0$ together with the valid relation (10):

$$
\frac{L_{\mathrm{J}}^{2}(\varrho)}{a^{3}} \Delta_{q} w=\frac{\varrho_{\mathrm{H}} \beta^{\prime}(\varrho)}{a^{2} \varrho} \Delta_{q} P .
$$

The coefficient in front of the (Eulerian) Laplacian of $\boldsymbol{P}$ is density-independent for the special choice $p=\kappa \varrho^{2}, \kappa=$ const., which corresponds to the assumption of an isolated and "virialized" fluid element, cf. Buchert \& Domínguez (2005, Appendix C). For this example we obtain an evolution equation of the form:

$$
\ddot{\boldsymbol{P}}+2 H \dot{\boldsymbol{P}}-4 \pi G \varrho_{\mathrm{H}} \boldsymbol{P}=\frac{2 \kappa \varrho_{\mathrm{H}}}{a^{2}} \Delta_{q} \boldsymbol{P}
$$

Note that the above model equation is nonlinear in Eulerian space (due to the convective non-linearities hidden in the overdot) and in Lagrangian space (the Eulerian Laplacian, if transformed to Lagrangian coordinates is non-linear). It is of a hybrid Lagrangian/Eulerian type and has been suggested in Buchert \& Domínguez (2005).

4 Linearization of the convective non-linearities in Eq. (12) yields the Eulerian linear approximation (e.g., Peebles 1980). For this, note that linearization of the general integral for the density (see Eq. (20) below) gives $\delta=-\nabla_{0} \cdot \boldsymbol{P}$.

5 By linearizing the Eulerian Laplacian in Eq. (12), now in the Lagrangian frame, i.e., retaining only the zero-order Lagrangian term $\boldsymbol{q}=\boldsymbol{X}+\boldsymbol{P} \approx \boldsymbol{X}$, we recover the Lagrangian linear approximation for a medium supported by a dynamical pressure (Adler \& Buchert 1999).

6 Restricting the latter model further by assuming that $\boldsymbol{u} \propto \boldsymbol{w}$, with the function of proportionality taken from the Eulerian linear approximation for a dust continuum, we obtain the standard adhesion approximation (Gurbatov et al. 1989).

7 The adhesion approximation reduces, for $L_{\mathrm{J}}=0$, to the Zel'dovich approximation (Zel'dovich 1970, 1973).

\subsection{Discussion of models}

While models 2 and 4-7 are perturbative, model 3 already describes the non-perturbative regime concerning the dynamical stress tensor. This non-perturbative approximation extrapolates the Lagrangian linear model by the replacement $\Delta_{X} \rightarrow \Delta_{X+P}$.

There are other models that are not covered by this list, notably higher-order perturbative approximations. For example, in the Lagrangian perturbation framework that contains the Zel'dovich approximation as a special first-order solution in a subclass (Buchert 1989, 1992), solutions have been derived for dust up to the fourth order. Special classes of second-order solutions and their investigation may be found in Bouchet et al. (1992, 1995) and Buchert \& Ehlers (1993). To the third order Moutarde et al. (1991) gave a special solution, Buchert (1994) and Catelan (1995) investigated a large class. Vanselow (1996) derived second- and third-order solutions for some more general cases, as well as a class of fourth-order solutions. By including isotropic stresses, Lagrangian perturbation solutions were given to first order (Adler \& Buchert 1999), to second order (Morita \& Tatekawa 2001; Tatekawa et al. 2002), and to third order (Tatekawa 2005a,b). All of these higher-order models can be regarded as approximating the residual vector field $\mathcal{R}$. However, in all of these models, the peculiar-gravitational field strength remains smooth while crossing high-density regions, which (as becomes clear below) points to a shortcoming of the perturbative calculations. The difficulty in deriving a closed differential equation for the displacement field $\boldsymbol{P}$ in the highly non-linear regime lies precisely in the term $\mathcal{R}$, which is a non-local (in space and time) and a non-linear functional of $\boldsymbol{P}$; approximating $\mathcal{R}$ perturbatively hides an important effect, as we shall see in detail below.

Finding an extension into the non-perturbative (both Eulerian and Lagrangian) regimes is an involved mathematical task. Notwithstanding, it should be attempted. For example, even the Lagrangian perturbation approach falls short capturing the action of multi-stream forces. This was demonstrated by comparing the statistical properties of second- and third-order Lagrangian perturbation solutions with results of numerical simulations (Tatekawa 2004a,b). This shortcoming calls for a nonperturbative generalization.

A proper understanding of the extrapolation of the linear relationship (10) between $\boldsymbol{w}$ and $\boldsymbol{P}$ into the non-perturbative regime requires that the residual term $\mathcal{R}$ in Eq. (6a) be analyzed in general. To this end we exploit the fact that Eq. (6a) holds independently of whether we are talking about dust or a general dispersion-supported system. It is possible to find exact integrals of the general Eq. (6a), which will also help us to understand the quality of the relationship (10) - lying at the basis of most currently known models - without involving higher-order perturbation analysis.

Finally, although virialized states can be understood through the tensor virial theorem, eventually including surface terms to account for the non-isolated state of gravitational systems, there are signatures possibly imprinted onto the phase space distribution during the non-perturbative regime. This distribution may have a "relaxed" global shape, but its internal structure will probably appear structured, rather than completely smooth, e.g. as a result of a hierarchy of embedded (smoothed) caustics (Ed Bertschinger, priv. comm.).

\section{Exact integral for the peculiar-gravitational field strength}

In this section we look at the general equations and combine them in order to obtain a transport equation for the peculiargravitational field strength. We then integrate the transport equation exactly with the help of a (sufficient) restricting condition. 
With this result we are able to calculate the gravitational field strength for a given family of trajectories, and to solve the longitudinal field equation $\nabla_{\boldsymbol{q}} \cdot \boldsymbol{w}=-4 \pi G \varrho_{\mathrm{H}} a \delta$ in general. We later demonstrate that this integral can be exploited to obtain a powerful approximation for the non-perturbative regime of structure formation.

\subsection{Transport equation for the peculiar-gravitational field strength}

We start with Eq. (2) and employ the vector identity

$$
\begin{aligned}
& \left(\boldsymbol{u} \cdot \nabla_{\boldsymbol{q}}\right) \boldsymbol{w}-\boldsymbol{u}\left(\nabla_{\boldsymbol{q}} \cdot \boldsymbol{w}\right)= \\
& \quad\left[\left(\boldsymbol{w} \cdot \nabla_{\boldsymbol{q}}\right) \boldsymbol{u}-\boldsymbol{w}\left(\nabla_{\boldsymbol{q}} \cdot \boldsymbol{u}\right)\right]+\nabla_{\boldsymbol{q}} \times(\boldsymbol{w} \times \boldsymbol{u})
\end{aligned}
$$

to find the modified evolution equation:

$$
\begin{aligned}
& \frac{\mathrm{d}}{\mathrm{d} t} \boldsymbol{w}+2 H \boldsymbol{w}-4 \pi G \varrho_{\mathrm{H}} \boldsymbol{u}= \\
& \quad \frac{1}{a}\left[\left(\boldsymbol{w} \cdot \nabla_{\boldsymbol{q}}\right) \boldsymbol{u}-\boldsymbol{w}\left(\nabla_{\boldsymbol{q}} \cdot \boldsymbol{u}\right)+\nabla_{\boldsymbol{q}} \times \tilde{\boldsymbol{T}}\right],
\end{aligned}
$$

with a new vector potential $\tilde{\boldsymbol{T}}:=\boldsymbol{T}+\boldsymbol{w} \times \boldsymbol{u}$. Computing $\varrho \frac{\mathrm{d}}{\mathrm{d} t}(\boldsymbol{w} / \varrho)$, and using the continuity Eq. (1a), we arrive at the following equation that we may call transport equation for $w$ :

$$
\begin{aligned}
& \frac{\mathrm{d}}{\mathrm{d} t}\left(\frac{\boldsymbol{w}}{\varrho}\right)-H\left(\frac{\boldsymbol{w}}{\varrho}\right)= \\
& \left(\frac{w}{\varrho} \cdot \nabla_{\boldsymbol{q}}\right) \frac{\boldsymbol{u}}{a}+4 \pi G \varrho_{\mathrm{H}} \frac{\boldsymbol{u}}{\varrho}+\frac{1}{a \varrho} \nabla_{\boldsymbol{q}} \times \tilde{\boldsymbol{T}} .
\end{aligned}
$$

This is the key-equation of the present work.

\subsection{Integrating the transport equation}

For the case $\nabla_{q} \times \tilde{\boldsymbol{T}}=\mathbf{0}$, we can find an exact integral to the above transport equation along comoving trajectories $\boldsymbol{q}=\boldsymbol{F}(\boldsymbol{X}, t)$ as follows.

Motivated by a recent investigation of an exact Lagrangian integral for the gravitational field strength $\boldsymbol{g}$ in Newtonian gravity (Buchert 2006a), we make the following ansatz (which generalizes the ansatz for the case of a non-vanishing background source in the above-mentioned work):

$$
\frac{\boldsymbol{w}-\zeta \boldsymbol{F}}{\varrho}=a\left(\boldsymbol{k} \cdot \nabla_{0}\right) \boldsymbol{F} ; \quad \zeta=\zeta(t) ; \quad \boldsymbol{k}=\boldsymbol{k}(\boldsymbol{X}),
$$

with the nabla-operator with respect to Lagrangian coordinates $\nabla_{0}$. Performing the total time-derivative of this ansatz along the integral curves $\boldsymbol{F}=\boldsymbol{X}+\boldsymbol{P}$ of the scaled peculiar-velocity field, i.e., $\frac{\mathrm{d}}{\mathrm{d} t} \boldsymbol{F}=\frac{1}{a} \boldsymbol{u}(\boldsymbol{F}, t)$, using a further identity (e.g., Serrin 1959),

$\left(\boldsymbol{k} \cdot \nabla_{0}\right) \frac{\mathrm{d}}{\mathrm{d} t} \boldsymbol{F}=\left[\left(\boldsymbol{k} \cdot \nabla_{0}\right) \boldsymbol{F}\right] \cdot \nabla_{\boldsymbol{q}} \frac{\boldsymbol{u}}{a}$,

and once more applying the identity (13) to the fields $\boldsymbol{u}$ and $\boldsymbol{F}$,

$$
\begin{aligned}
& \nabla_{\boldsymbol{q}} \cdot\left[\boldsymbol{F}\left(\nabla_{\boldsymbol{q}} \cdot \boldsymbol{u}\right)-\left(\boldsymbol{F} \cdot \nabla_{\boldsymbol{q}}\right) \boldsymbol{u}\right]= \\
& \nabla_{\boldsymbol{q}} \cdot\left[\boldsymbol{u}\left(\nabla_{\boldsymbol{q}} \cdot \boldsymbol{F}\right)-\left(\boldsymbol{u} \cdot \nabla_{\boldsymbol{q}}\right) \boldsymbol{F}\right]=\nabla_{\boldsymbol{q}} \cdot(d-1) \boldsymbol{u},
\end{aligned}
$$

(with $d$ denoting the dimension of space), we obtain an equation that we compare with the transport equation. We are left with the following conditions on the unknown function $\zeta$ :

$$
\frac{\boldsymbol{F}}{\varrho}(\dot{\zeta}+2 H \zeta)=\mathbf{0} ; \quad \frac{\boldsymbol{u}}{\varrho}\left(\zeta-\frac{4 \pi G_{\mathrm{H}} a}{d}\right)=\mathbf{0} .
$$

For non-vanishing $\boldsymbol{F}$ and $\boldsymbol{u}$, the above two equations for $\zeta$ are equivalent by virtue of $\dot{\varrho}_{\mathrm{H}}=-3 H \varrho_{\mathrm{H}}$, and we have determined the unkown function:

$\zeta=\frac{4 \pi G \varrho_{\mathrm{H}} a}{d}$

Now, we can write down an exact integral $\boldsymbol{w}^{I}$ for the peculiargravitational field-strength. We also replace the density by its exact Lagrangian integral,

$\varrho(\boldsymbol{X}, t)=\frac{\varrho\left(\boldsymbol{X}, t_{0}\right)}{J}=\varrho_{\mathrm{H}} \frac{1+\delta\left(\boldsymbol{X}, t_{0}\right)}{J_{\mathrm{F}}} ; \varrho_{\mathrm{H}}=\frac{\varrho_{\mathrm{H}}\left(t_{0}\right)}{a^{3}}$,

where $J:=\operatorname{det}\left(f_{i \mid k}\right)$ denotes the Jacobian of the transformation from $\boldsymbol{x}=\boldsymbol{f}(\boldsymbol{X}, t)$ to $\boldsymbol{X}$, and $J_{\mathrm{F}}:=\operatorname{det}\left(F_{i \mid k}\right)=J a^{-3}$ denotes the Jacobian of the transformation from $\boldsymbol{q}=\boldsymbol{F}(\boldsymbol{X}, t)$ to $\boldsymbol{X}$ with the comoving Lagrangian deformation gradient $\left.\left(F_{i \mid k}\right)^{5}\right)$. The result reads

$\boldsymbol{w}^{I}=\frac{\left(\boldsymbol{K} \cdot \nabla_{0}\right) \boldsymbol{F}}{a^{2} J_{\mathrm{F}}}+\frac{4 \pi G \varrho_{\mathrm{H}} a}{d} \boldsymbol{F} ;$

$\boldsymbol{K}=\frac{\boldsymbol{k}}{\varrho\left(t_{0}\right)}=\boldsymbol{W}-\frac{4 \pi G \varrho_{\mathrm{H}}\left(t_{0}\right)}{d} \boldsymbol{X}$ is the integration constant, which was determined by evaluating the integral at $t=t_{0}$. The integral (21) explicitly depends on the dimension $d$ of the continuum.

The above integral is quasi-local, i.e. it locally represents the peculiar-gravitational field strength through a functional of the comoving trajectory field $\boldsymbol{F}$, while initial data are constructed non-locally according to the structure of the theory.

A strong property of the integral (21) is that it solves the longitudinal field equation $\nabla_{\boldsymbol{q}} \cdot \boldsymbol{w}=-4 \pi G \varrho_{\mathrm{H}} a \delta$ in general. (We can also find such an integral for the remaining field equations $\nabla_{\boldsymbol{q}} \times \boldsymbol{w}=\mathbf{0}$, see Sect. 5; a general integral is expected to include non-local terms.)

\subsection{Lagrangian framework, proof of the exact integral and its transformation properties}

Let us recall the basic equations of a Lagrangian description of fluid motion. As shown in Buchert (2006a), the presented integral (21) can also be obtained by simply transforming the result obtained in Newtonian gravity. This is expected for exact equations and expressions. The explicit derivation in this paper shows that the approximation of a vanishing curl of the vector potential $\tilde{\boldsymbol{T}}$ apparently does not impair this property; in general, approximations to the system of equations in Newtonian gravity do not carry over to corresponding approximations in Newtonian cosmology by a simple transformation.

In Newtonian gravity the Lagrangian description is based on integral curves $\boldsymbol{x}=\boldsymbol{f}(\boldsymbol{X}, t)$ of the full velocity field $\boldsymbol{v}(\boldsymbol{x}, t)$ :

$\frac{\mathrm{d} \boldsymbol{f}}{\mathrm{d} t}=\boldsymbol{v}(\boldsymbol{f}, t) ; \boldsymbol{f}\left(\boldsymbol{X}, t_{0}\right)=: \boldsymbol{X}$.

By introducing this family of trajectories, we can express all Eulerian fields (e.g., the velocity $\boldsymbol{v}$, the acceleration $\boldsymbol{g}$, the density $\varrho$, and the vorticity $\left.\omega:=\frac{1}{2} \nabla_{x} \times \boldsymbol{v}\right)$ in terms of the field of trajectories $\boldsymbol{x}=\boldsymbol{f}(\boldsymbol{X}, t)$ as follows:

$v=\dot{f}(X, t) ; \quad g=\ddot{f}(X, t) ;$

$\varrho=\frac{\varrho_{0}}{J} ; \quad \omega=\frac{\omega_{0} \cdot \nabla_{0} f}{J}$,

5 We denote a spatial derivative with respect to Lagrangian coordinates by a vertical slash | that commutes with the Lagrangian timederivative. 
with the Jacobian of the transformation from Eulerian to Lagrangian coordinates $J:=\operatorname{det}\left(f_{i \mid k}(\boldsymbol{X}, t)\right)>0, \varrho_{0}:=\varrho\left(\boldsymbol{X}, t_{0}\right)$, vec $\omega_{0}:=\omega\left(X, t_{0}\right)$.

Equation (24) lists the known Lagrangian integrals for the density $\varrho$ and the vorticity $\omega$ of the Euler-Newton system; i.e., they represent a Eulerian field as a functional of $f$. To transform those fields back to Eulerian space, we need the transformation $f$ to be invertible; i.e. $J>0$, defining regular solutions (for more details the reader may consult the review by Ehlers \& Buchert 1997).

The Eulerian field equations are transformed into a system of Lagrangian equations by virtue of the following transformation of the field strength gradient:

$\frac{\partial}{\partial x_{j}} g_{i}=g_{i \mid k} \frac{\partial}{\partial x_{j}} h_{k}=\frac{1}{2 J} \epsilon_{k \ell m} \epsilon_{j p q} g_{i \mid k} f_{p \mid \ell} f_{q \mid m}$.

The gradient of the inverse transformation from Lagrangian to Eulerian coordinates, $\boldsymbol{h}=\boldsymbol{f}^{-1}$, was expressed in terms of $\boldsymbol{f}$ through the algebraic relationship

$\frac{\partial}{\partial x_{j}} h_{i}=J_{i j}^{-1}=\operatorname{ad}\left(J_{i j}\right) J^{-1}=\frac{1}{2 J} \epsilon_{i k \ell} \epsilon_{j m n} f_{m \mid k} f_{n \mid \ell}$.

For the field equations we obtain with (25) the following set of four Lagrangian equations (Buchert \& Götz 1987 for $\Lambda=0$, and Buchert 1989 for $\Lambda \neq 0)(i, j, k=1,2,3$ with cyclic ordering; summation over repeated indices is understood):

$\frac{1}{2} \epsilon_{a b c} \frac{\partial\left(g_{a}, f_{b}, f_{c}\right)}{\partial\left(X_{1}, X_{2}, X_{3}\right)}-\Lambda J=-4 \pi G \varrho_{0}(\boldsymbol{X}) ;$

$\epsilon_{p q[j} \frac{\partial\left(g_{i]}, f_{p}, f_{q}\right)}{\partial\left(X_{1}, X_{2}, X_{3}\right)}=0, i \neq j$.

In the case of a dust continuum, the above equations form a set of four evolution equations, the Lagrange-Newton system of equations, by virtue of $\boldsymbol{g}=\ddot{\boldsymbol{f}}$. Alternative forms of these equations may be found in Buchert (1996) and Ehlers \& Buchert (1997).

The above equations not only hold for a continuum of dust; they simply represent a transformation of the field equations, which also hold in the more general setting discussed in Sect. 2. The difference comes in when we represent the field strength $g$ in terms of the trajectory field; e.g. including isotropic pressure forces the resulting Lagrangian evolution equations are investigated in Adler \& Buchert (1999).

Proposition 2 in Buchert (2006a) gives the following integral of the first Lagrangian equation (including its proof):

$\boldsymbol{g}^{I \Lambda}=\left(\boldsymbol{C} \cdot \nabla_{0}\right) \boldsymbol{f} J^{-1}+\frac{\Lambda}{d} \boldsymbol{f}, \boldsymbol{C}:=\boldsymbol{G}-\frac{\Lambda}{d} \boldsymbol{X}$.

Since this result is exact, we are entitled to apply the transformation to comoving coordinates and peculiar-fields to this result. Since we can adopt the same Lagrangian coordinates $\boldsymbol{X}$ in both cases in view of $\boldsymbol{x}=a(t) \boldsymbol{q}, a\left(t_{0}\right)=1$ and since the background field strength evolves as $\boldsymbol{g}_{\mathrm{H}}=\ddot{a} \boldsymbol{q}$, we make the ansatz $\boldsymbol{g}=\ddot{a} \boldsymbol{F}+\boldsymbol{w}(\boldsymbol{X}, t), \boldsymbol{G}=\ddot{a}\left(t_{0}\right) \boldsymbol{X}+\boldsymbol{W}(\boldsymbol{X})$ and use Friedmann's equation $3 \ddot{a}=\Lambda-4 \pi G a \varrho_{\mathrm{H}}$ to obtain the integral $\boldsymbol{w}^{I}$ in Eq. $(21)^{6}$.

\section{The exact integral and its implications}

In order to learn more about the implications of the integral (21), we now recover known model equations in cosmology (e.g. the Lagrangian perturbation scheme; this should be possible for the longitudinal parts, since the integral (21) is general in this case). Then, we elaborate on possible applications.

${ }^{6}$ For an independent proof we could instead insert (21) into the transformed Lagrangian equations given in Appendix A of Buchert (1989).

\subsection{Recovering known cosmological models}

We first write the general expression for $\boldsymbol{w}$ in terms of $\boldsymbol{P}$. In the case of a dust continuum, $\boldsymbol{w}=a(\ddot{\boldsymbol{P}}+2 H \dot{\boldsymbol{P}})$ (from Euler's equation for dust $\boldsymbol{w}=\dot{\boldsymbol{u}}+H \boldsymbol{u}$ with $\boldsymbol{u}=a \dot{\boldsymbol{P}}$ ), we rewrite the integral (21) in terms of $\boldsymbol{P}$ (we drop the index $I$ to ease the notation, consider the case $d=3$ and divide by $a$ ):

$\ddot{\boldsymbol{P}}+2 H \dot{\boldsymbol{P}}=\frac{\left(\boldsymbol{K} \cdot \nabla_{0}\right)(\boldsymbol{X}+\boldsymbol{P})}{a^{3} J_{\mathrm{F}}}+\frac{4 \pi G \varrho_{\mathrm{H}}}{3}(\boldsymbol{X}+\boldsymbol{P})$,

with the following general expresssion for the Jacobian $J_{\mathrm{F}}=a^{-3} J$ :

$J_{\mathrm{F}}=1+I\left(P_{i \mid k}\right)+I I\left(P_{i \mid k}\right)+I I I\left(P_{i \mid k}\right)$,

where $I, I I$ and $I I I$ denote the principal scalar invariants of the tensor in brackets.

Let us first expand the expression $1 / J_{\mathrm{F}}$ with the Jacobian (31) to first order, $1 / J_{\mathrm{F}}=1 /\left(1+\nabla_{0} \cdot \boldsymbol{P}+\ldots\right) \approx 1-\nabla_{0} \cdot \boldsymbol{P}$. We insert $\boldsymbol{K}=: \boldsymbol{W}-\frac{4 \pi G \varrho_{\mathrm{H}}\left(t_{0}\right)}{3} \boldsymbol{X}$, drop terms that are quadratic in the fields $\boldsymbol{P}$ and $\boldsymbol{W}$, and replace the term $\left(\boldsymbol{X} \cdot \nabla_{0}\right) \boldsymbol{P}-\boldsymbol{X}\left(\nabla_{0} \cdot \boldsymbol{P}\right)$ by the (up to a transversal part equivalent) term $\left(\boldsymbol{P} \cdot \nabla_{0}\right) \boldsymbol{X}-\boldsymbol{P}\left(\nabla_{0} \cdot \boldsymbol{X}\right)=-2 \boldsymbol{P}$. This approximated equation then reads:

$\ddot{\boldsymbol{P}}^{(1)}+2 H \dot{\boldsymbol{P}}^{(1)}-4 \pi G \varrho_{\mathrm{H}} \boldsymbol{P}^{(1)}=\frac{\boldsymbol{W}}{a^{3}}$.

Note that the factor 3 disappeared from the term in front of $\boldsymbol{P}^{(1)}$. This is the equation for longitudinal first-order Lagrangian perturbations (Buchert 1989, 1992). For initial quasi-homogeneity, which is sometimes assumed, $c f$. Footnote 4, the right-hand side of this equation drops.

Expanding $1 / J_{\mathrm{F}}$ to higher orders soon yields messy expressions; e.g. by keeping only terms quadratic in $\boldsymbol{P}^{(1)}$ and $\boldsymbol{W}$, we deal with an approximate equation of the form

$$
\begin{aligned}
\ddot{\boldsymbol{P}}^{(2)}+2 H \dot{\boldsymbol{P}}^{(2)} & -4 \pi G \varrho_{\mathrm{H}} \boldsymbol{P}^{(2)}=\frac{\boldsymbol{W}}{a^{3}} \\
& +\frac{1}{a^{3}}\left(\boldsymbol{W} \cdot \nabla_{0} \boldsymbol{P}^{(1)}-\boldsymbol{W} \nabla_{0} \cdot \boldsymbol{P}^{(1)}\right)+\cdots
\end{aligned}
$$

The last term in the above equation illustrates that we recover source terms for the second-order perturbation solution $\boldsymbol{P}^{(2)}$, which correspond to the local parts (as introduced in Buchert 1993; Buchert 1994); the full terms including the non-local parts are solutions of Poisson equations with sources given by the divergence of expressions of this type.

By expressing $\boldsymbol{w}$ through more general Euler equations that include presssure terms, velocity dispersion, or deviations from mean field gravity (cf. Buchert \& Domínguez 2005), we can also expand the integral in order to recover known approximations, e.g., the first-order Lagrangian equation for longitudinal perturbations in a medium supported by isotropic pressure forces (Adler \& Buchert 1999).

\subsection{Iteration approach}

A powerful possibility of applying the exact integral focusses on an iterative definition of the trajectories or of the displacement field. This iterative view exploits the fact that we know the field strength exactly (and in general with respect to the longitudinal field equation) along any trajectory field that we could imagine.

We now exemplify the iteration procedure for a continuum of dust. Note that the integral (21) can be viewed as a set of three partial differential equations for the trajectory field after inserting the general expression for the peculiar-acceleration field, e.g. 
for dust: $\boldsymbol{w}=a(\ddot{\boldsymbol{F}}+2 H \dot{\boldsymbol{F}})$. Holding the Lagrangian coordinates fixed, i.e. for a single trajectory, we are dealing with ordinary differential equations.

The way we define the iteration scheme enjoys some freedom (non-unique definition). We argue below for the following choice of writing the integral (21) as an iteration scheme:

$\frac{1}{a} \boldsymbol{w}^{[n+1]}-4 \pi G \varrho_{\mathrm{H}} \boldsymbol{F}^{[n+1]}=\frac{\left(\boldsymbol{K} \cdot \nabla_{0}\right) \boldsymbol{F}^{[n]}}{a^{3} J_{\mathrm{F}}^{[n]}}-\frac{8 \pi G \varrho_{\mathrm{H}}}{3} \boldsymbol{F}^{[n]}$,

with $\boldsymbol{K}$ given in (21), and $\frac{1}{a} \boldsymbol{w}^{[n+1]}=\ddot{\boldsymbol{F}}^{[n+1]}+2 H \dot{\boldsymbol{F}}^{[n+1]}$, denoting the iteration steps in brackets to avoid confusion with the perturbation index used earlier. In this way we have split the linear term $\frac{4 \pi G \varrho_{\mathrm{H}}}{d} \boldsymbol{F}$ for $d \equiv 3$ into $4 \pi G \varrho_{\mathrm{H}}-\frac{2}{3} 4 \pi G \varrho_{\mathrm{H}}$, the first term we consider as belonging to $[n+1]$ and the second to $[n]$. The reason for this choice becomes obvious after the remarks below.

For the displacement field $\boldsymbol{P}=\boldsymbol{F}-\boldsymbol{X}$, we obtain the following iteration scheme:

$$
\begin{aligned}
\ddot{\boldsymbol{P}}^{[n+1]}+2 H \dot{\boldsymbol{P}}^{[n+1]}-4 \pi G \varrho_{\mathrm{H}} \boldsymbol{P}^{[n+1]}= \\
\quad \frac{4 \pi G \varrho_{\mathrm{H}}}{3}\left(\boldsymbol{X}-2 \boldsymbol{P}^{[n]}\right)+\frac{\left(\boldsymbol{K} \cdot \nabla_{0}\right)\left(\boldsymbol{X}+\boldsymbol{P}^{[n]}\right)}{a^{3} \operatorname{det}\left(\delta_{i k}+P_{i \mid k}^{[n]}\right)} .
\end{aligned}
$$

Iteration consists in the strategy of feeding in a comoving displacement field $\boldsymbol{P}^{[n]}$ on the right-hand side of Eq. (35) in order to obtain another displacement field $\boldsymbol{P}^{[n+1]}$ by solving secondorder, ordinary differential equations for each fluid element $\boldsymbol{X}$. The above choice of the iteration scheme is motivated by three arguments. First, starting the iteration (34) with the trivial trajectory field $\boldsymbol{F}^{[0]}=\boldsymbol{X}$, which corresponds to a straight Hubble expansion, we obtain the equation governing the longitudinal first-order Lagrangian approximation exactly, $\boldsymbol{F}^{[1]}=\boldsymbol{X}+\boldsymbol{P}^{[1]}$, with $\boldsymbol{P}^{[1]}$ obeying Eq. (32) for $\boldsymbol{P}^{(1)}$. Second, the choice is supported by the fact that $\boldsymbol{F}^{[0]}$ (as a special exact solution of the Lagrange-Newton system $\{27,28\}$ ) also produces a special exact solution $\boldsymbol{F}^{[1]}$ of the same system (Buchert 1989). Third, to all orders in Lagrangian perturbation theory, the longitudinal differential operators for $\boldsymbol{P}^{(n+1)}$ have all the form of the differential operator for $\boldsymbol{P}^{(1)}$, Eq. (32), with sources involving the lower-order perturbation solutions (see Ehlers \& Buchert 1997, Sect. 3.2, for the general perturbation and solution schemes); compare with the left-hand side of Eq. (35).

However, this does not mean that further iteration eventually produces further exact solutions, and also that another choice could not perform as well as the above choice. In order to illustrate the iteration scheme further, we compute the equation for the second iterate $\boldsymbol{P}^{[2]}$. While the first iterate (insert $\boldsymbol{P}^{[0]}=\mathbf{0}$ into Eq. (35)) obeys

$\ddot{\boldsymbol{P}}^{[1]}+2 H \dot{\boldsymbol{P}}^{[1]}-4 \pi G \varrho_{\mathrm{H}} \boldsymbol{P}^{[1]}=\frac{\boldsymbol{W}}{a^{3}}$,

the second iterate may be found by inserting only a subclass of the general solution to Eq. (36) that corresponds to Zel'dovich's approximation (Zel'dovich 1970, 1973). After factorizing $\boldsymbol{P}^{[1]}$ into time-dependent functions and vector-functions of initial data, the time-dependent solutions consist of two homogeneous and one particular solution of the following equation (Buchert 1992):

$\ddot{\xi}(t)+2 H(t) \dot{\xi}(t)-4 \pi G \varrho_{\mathrm{H}}(t)(\xi(t)+1)=0$,

where $H(t)=\frac{\dot{a}}{a}$ and $\varrho_{\mathrm{H}}(t)=\varrho_{\mathrm{H}}\left(t_{0}\right) a^{-3}$ have to be expressed through solutions of Friedmann's differential equation:

$\frac{\dot{a}^{2}}{a^{2}}-\frac{8 \pi G \varrho_{\mathrm{H}}}{3}+\frac{k}{a^{2}}-\frac{\Lambda}{3}=0$.
Explicit forms of the functions $\xi(t)$ including a cosmological constant can be found in (Bildhauer et al. 1992; see also the supplement by Chernin et al. 2003). We choose the restricted initial data set for which $\boldsymbol{U} \propto \boldsymbol{W}\left(\boldsymbol{U}(\boldsymbol{X}):=\boldsymbol{u}\left(\boldsymbol{q}, t_{0}\right)\right.$ ) (for more details see: Buchert 1992). Inserting this subclass of first-order solutions (Zel'dovich's approximation), $\boldsymbol{P}^{[1 Z]}:=b(t) \nabla_{0} \Psi(\boldsymbol{X})$, with the growing mode solution $\xi_{1}(t)=: b(t)$ of Eq. (37a), we can find the second iterate $\boldsymbol{P}^{[2 Z]}$ corresponding to this restricted choice as follows (we also use expression (31)):

$$
\begin{gathered}
\ddot{\boldsymbol{P}}^{[2 Z]}+2 H \dot{\boldsymbol{P}}^{[2 Z]}-4 \pi G \varrho_{\mathrm{H}} \boldsymbol{P}^{[2 Z]}= \\
\frac{4 \pi G \varrho_{\mathrm{H}}}{3}\left(\boldsymbol{X}-2 b(t) \nabla_{0} \Psi(\boldsymbol{X})\right) \\
+\frac{\left(\boldsymbol{K} \cdot \nabla_{0}\right)\left(\boldsymbol{X}+b(t) \nabla_{0} \Psi(\boldsymbol{X})\right)}{a^{3}\left[1+b I\left(\Psi_{\mid i k}\right)+b^{2} I I\left(\Psi_{\mid i k}\right)+b^{3} I I I\left(\Psi_{\mid i k}\right)\right]} .
\end{gathered}
$$

This equation defines a set of ordinary differential equations parametrized by $\boldsymbol{X}$. Its analytical solution (the subject of a forthcoming work) provides a test case for a numerical iteration scheme. For this purpose we now give some useful technicalities.

\subsection{Numerical implementation}

For the purpose of numerically implementing the iteration scheme (35), we may rescale the dependent and independent variables as follows. Following Shandarin (1980) except that we refer all quantities with subscript " 0 " to the initial time $t_{0}$, we introduce the dimensionless and appropriately scaled variables (Buchert 1989, Appendix A)

$\tilde{\boldsymbol{q}}:=\boldsymbol{q} / q_{0} ; \tilde{\boldsymbol{u}}:=\left(\boldsymbol{u} / u_{0}\right) a ; \tilde{\boldsymbol{w}}:=\left(\boldsymbol{w} / w_{0}\right) a^{3}$,

$u_{0}=q_{0} / t_{0} ; \quad w_{0}=q_{0} / t_{0}^{2} ; \quad \tilde{\varrho}:=\left(\varrho / \varrho_{\mathrm{H}}\left(t_{0}\right)\right) a^{3}$,

and a conformal transformation of the time-variable:

$\mathrm{d} T:=\frac{1}{t_{0}} \frac{\mathrm{d} t}{a^{2}(t)}$

which is negative and tends to $-\infty$ at the Big-Bang singularity. With the help of (39b), we can write the solutions of Friedmann's differential Eq. (37b) and the mass density parameter for the cases $\Lambda=0^{7}$ in simple forms:

$a(T)=\frac{T_{0}^{2}+k}{T^{2}+k} ; \quad \Omega^{m}:=\frac{8 \pi G \varrho_{\mathrm{H}}}{3 H^{2}}=\frac{T^{2}+k}{T^{2}}$,

with $k=0, \pm 1$. For an Einstein-de Sitter model $(k=0)$, we have $T_{0}=-3$, and for the other models $T_{0}=-\sqrt{\frac{k}{\Omega_{0}^{m}-1}}$. Below we also use the relations $4 \pi G \varrho_{\mathrm{H}}\left(t_{0}\right)=\frac{3}{2} \Omega_{0}^{m} H_{0}^{2}$ and $4 \pi G \varrho_{\mathrm{H}}\left(t_{0}\right) t_{0}^{2}=$ $6 /\left(T_{0}^{2}+k\right)$

(for further details see: Buchert 1989, Appendix A).

For the scaled displacement field,

$\tilde{\boldsymbol{P}}:=\frac{\boldsymbol{P}}{q_{0}} ; \quad \tilde{\boldsymbol{X}}:=\frac{\boldsymbol{X}}{q_{0}} ; \quad \tilde{\nabla}_{\mathbf{0}}=q_{0} \nabla_{0}$,

Eq. (35) can be rewritten as a set of first-order (for each $\tilde{\boldsymbol{X}}$ ordinary) differential equations:

$$
\begin{gathered}
\frac{\mathrm{d}}{\mathrm{d} T} \tilde{\boldsymbol{P}}^{[n+1]}=\tilde{\boldsymbol{u}}^{[n+1]} \\
\frac{T^{2}+k}{2} \frac{\mathrm{d}}{\mathrm{d} T} \tilde{\boldsymbol{u}}^{[n+1]}-3 \tilde{\boldsymbol{P}}^{[n+1]}= \\
\left(\tilde{\boldsymbol{X}}-2 \tilde{\boldsymbol{P}}^{[n]}\right)+\frac{\left(\tilde{\boldsymbol{K}} \cdot \tilde{\nabla}_{\mathbf{0}}\right)\left(\tilde{\boldsymbol{X}}+\tilde{\boldsymbol{P}}^{[n]}\right)}{\operatorname{det}\left(\delta_{i k}+\tilde{P}_{i \mid k}^{[n]}\right)}
\end{gathered}
$$

${ }^{7}$ For the cases $\Lambda \neq 0$ we have to employ other strategies, e.g.: Bildhauer et al. (1992). 
with $\tilde{\boldsymbol{K}}:=\frac{1}{2}\left(T_{0}^{2}+k\right) \tilde{\boldsymbol{W}}-\tilde{\boldsymbol{X}}$. The differential operator in the second equation simplifies further by integrating along the time $\tau$ with $\mathrm{d} \tau:=2 a(T) /\left(T_{0}^{2}+k\right) \mathrm{d} T$. Initial data for $\tilde{\boldsymbol{W}}$ follow from solving the initial Poisson equation for the density contrast $\delta=\tilde{\varrho}-1$ :

$\tilde{\nabla}_{\mathbf{0}} \cdot \frac{1}{2}\left(T_{0}^{2}+k\right) \tilde{\boldsymbol{W}}=-3 \delta(\tilde{\boldsymbol{X}}) ; \quad \tilde{\nabla}_{\mathbf{0}} \times \tilde{\boldsymbol{W}}=\mathbf{0}$.

Initial data for $\tilde{\boldsymbol{U}}$ could be specified by the special choice $\tilde{\boldsymbol{U}}=\tilde{\boldsymbol{W}}$.

As a first test of a numerical scheme one chooses $\tilde{\boldsymbol{P}}^{[0]}=\mathbf{0}$, so that $\tilde{\boldsymbol{P}}^{[1]}$ should be identical to Zel'dovich's approximation (Zel'dovich 1970, 1973). A second test will be provided by the analytical solution for the second iterate, as mentioned above. A third test should measure the (artificial) vorticity of $\boldsymbol{w}$ as introduced by the integral for generic initial data; here, one can compare with the exact expression (44) below for the transverse part of the field strength.

All peculiar-fields appearing in the calculations must be periodic on the largest scale to assure that the average model is Friedmannian. This construction is necessary for the uniqueness of a Newtonian solution and implies a globally vanishing "backreaction" (see: Buchert \& Ehlers 1997).

\section{Summary and prospects}

Current analytical models (excluding higher-order perturbative corrections) basically follow from the simple assumption that the peculiar-gravitational field strength is proportional to the displacement field, Eq. (10): $\boldsymbol{w}=\frac{W}{a^{2}}+4 \pi G \varrho_{\mathrm{H}} a \boldsymbol{P}$. Equation (12) furnishes the up to date most general model equation based on this assumption. More frequently employed models, like the celebrated Zel'dovich approximation and the adhesion approximation, even imply the tighter restriction of proportionality for the peculiar-gravitational field strength to the peculiar-velocity, which is a very good assumption in the weakly non-linear regime, but certainly fails in a highly non-linear situation. Consequences of the above remarks have been investigated in detail in (Buchert \& Domínguez 2005).

We have argued that relationship (10) needs generalization for the understanding of the non-perturbative regime of cosmic structure formation. Integrating the transport equation for the peculiar-gravitational field strength, Eq. (15), we obtained an exact integral, generalizing relationship (10). It was demonstrated that this integral can be employed to define an iteration scheme that allows us to obtain the peculiar-gravitational field strength for any given family of trajectories, which solves the Lagrangian evolution equation corresponding to $\nabla_{q} \cdot w=$ $-4 \pi G \varrho_{\mathrm{H}} a \delta$ in general. This property, together with the experience of the good performance of Lagrangian perturbation schemes based on the longitudinal part only (e.g., Buchert et al. 1997), supports the expectation that the integral and its corresponding iteration scheme provide a powerful approximation for the non-perturbative regime of structure formation.

The qualitative difference from a Lagrangian perturbation analysis is due to the fact that the former lacks the important leading term proportional to the density in (21). Lagrangian perturbation solutions predict a smooth gravitational field strength when crossing caustics in the density field for all orders in perturbation theory (compare the general perturbation and solution schemes given by Ehlers \& Buchert 1997). Therefore, we are entitled to consider the integral (21) as a genuinly non-perturbative result. It shows that a blow-up of the field strength at caustics is a generic property of the gravitational collapse. Counterarguments based on exact solutions with symmetry do not apply to the generic situation. Consider, as an example, plane-symmetric motions on a three-dimensional homogeneous-isotropic background. In that case the general exact solution (at the same time a solution of Eq. (32)) does not predict a singular field strength: specifying the integral (21) to plane-symmetric motion, we infer from the expression proportional to the density,

$$
\frac{\left(\left(\boldsymbol{W}-\frac{4 \pi G \varrho_{\mathrm{H}}\left(t_{0}\right)}{3} \boldsymbol{X}\right) \cdot \nabla_{0}\right)(\boldsymbol{X}+\boldsymbol{P})}{a^{2} J_{\mathrm{F}}},
$$

with $J_{\mathrm{F}}=1+P_{1 \mid 1}$,

that the Jacobian cancels the directional derivative term exactly, and $w_{1}^{\text {plane }}=4 \pi G \varrho_{\mathrm{H}} a P_{1}+W_{1} / a^{2}$ does not blow up at the caustic where $1+P_{1 \mid 1}=0$.

One of the most promising application fields of the integral and its iteration scheme could be the following. Since (21) provides $\boldsymbol{w}$ as a local functional of the displacement field $\boldsymbol{P}$, it may substantially enhance the power of reconstruction methods (Croft \& Gaztañaga 1997; Susperregi \& Buchert 1997; Courteau \& Willick 2000; Courteau \& Dekel 2001; Brenier et al. 2003; Mohayaee et al. 2003). As mentioned above, the integral (21) generalizes the assumption (10), which itself lies at the basis of most analytical models (as summarized in this paper) and which already furnishes a more general ansatz for reconstruction methods, compared to those that are currently implemented, e.g. those that assume the proportionality of $\boldsymbol{w}$ and $\boldsymbol{u}$.

To propose a non-perturbative approximation based on the integral (21) is also motivated by simplicity, besides the advantage of a local expression. On theoretical grounds, however, there are limitations to the integral (21), because it will produce artificial vorticity of $\boldsymbol{w}$, since a generic approximation based on (21) will not satisfy $\nabla_{\boldsymbol{q}} \times \boldsymbol{w}=\mathbf{0}$. Numerical work has to confirm the expectation that the longitudinal part of the field equations provides the dominant contribution. The transverse contribution and the contribution from non-local terms can also be quantified, based on results of a forthcoming work on transported differential forms associated with the gravitational field strength; we here already give the exact Lagrangian integral that solves the transverse field equation in general (Buchert 2006b):

$\boldsymbol{w}_{k}^{\text {transverse }}=\frac{1}{a J_{\mathrm{F}}}\left(W_{i} J_{i k}^{\mathrm{sub}}-\left(\boldsymbol{W} \times\left(\nabla_{0} \times \boldsymbol{F}\right) \cdot \nabla_{0} \boldsymbol{F}\right)_{k}\right)$,

where $J_{i k}^{\mathrm{sub}}$ denotes the subdeterminants of $\left(F_{i \mid k}\right)$.

Since a general integral, if it exists, would include non-local parts, and thus would require the solution of elliptic boundary value problems at all times, it may be of limited practical use.

Acknowledgements. This work was supported by the "Sonderforschungsbereich SFB 375 für Astro-Teilchenphysik der Deutschen Forschungsgemeinschaft". Thanks go to Stéphane Colombi for stimulating discussions and thoughts on the numerical implementation of the iteration scheme.

\section{References}

Adler, S., \& Buchert, T. 1999, A\&A, 343, 317

Bagla, J. S., \& Padmanabhan, T. 1997, Pramana, 49, 161 [arXiv: astro-ph/0411730]

Bernardeau, F., Colombi, S., Gaztañaga, E., \& Scoccimarro, R. 2002, Phys. Rep., 367,1

Bertschinger, E. 1998, ARA\&A, 36, 599

Bildhauer, S., Buchert, T., \& Kasai, M. 1992, A\&A, 263, 23

Binney, J., \& Tremaine, S. 1987, Galactic Dynamics (Princeton University Press) Bouchet, F. R., Juszkiewicz, R., Colombi, S., \& Pellat, R. 1992, ApJ, 394, L5

Bouchet, F. R., Colombi, S., Hivon, E., \& Juszkiewicz, R. 1995, A\&A, 296, 575 Brenier, Y., Frisch, U., Hénon, M., et al. 2003, MNRAS, 346, 501 
Buchert, T. 1989, A\&A, 223, 9

Buchert, T. 1992, MNRAS, 254, 729

Buchert, T. 1993, A\&A, 267, L51

Buchert, T. 1994, MNRAS, 267, 811

Buchert, T. 1996, in Proc. IOP Enrico Fermi, Course CXXXII (Dark Matter in the Universe), Varenna 1995, ed. S. Bonometto, J. Primack, \& A. Provenzale (IOS Press Amsterdam), 543

Buchert, T. 2006a, Phys. Lett. A, 354, 8

Buchert, T. 2006b, in preparation

Buchert, T., \& Domínguez, A. 1998, A\&A, 335, 395

Buchert, T., \& Domínguez, A. 2005, A\&A, 438, 443

Buchert, T., \& Ehlers, J. 1993, MNRAS, 264, 375

Buchert, T., \& Ehlers, J. 1997, A\&A, 320, 1

Buchert, T., \& Götz, G. 1987, J. Math. Phys., 28, 2714

Buchert, T., Karakatsanis, G., Klaffl, R., \& Schiller, P. 1997, A\&A, 318, 1

Catelan, P. 1995, MNRAS, 276, 115

Chernin, A. D., Nagirner, D. I., \& Starikova, S. V. 2003, A\&A, 399, 19

Courteau, S., \& Dekel, A. 2001, in Astrophysical Ages and Times Scales, ASP Conf. Ser., 245., ed. T. V. Hippel, C. Simpson, \& N. Manset

Courteau, S., \& Willick, J. 2000 (eds.), Cosmic Flows Workshop, ASP Conf. Ser., 201

Croft, R. A. C., \& Gaztañaga, E. 1997, MNRAS, 285, 793

Ehlers, J., \& Buchert, T. 1997, Gen. Rel. Grav., 29, 733

Gabrielli, A., Baertschiger, T., Joyce, M., Marcos, B., \& Sylos-Labini, F. 2006 [arXiv: cond-mat/0603124]

Gurbatov, S. N., Saichev, A. I., \& Shandarin, S. F. 1989, MNRAS, 236, 385

Ma, C.-P., \& Bertschinger, E. 2004, ApJ, 612, 28
Morita, M., \& Tatekawa, T. 2001, MNRAS, 328, 815

Mohayaee, R., Frisch, U., Matarrese, S., \& Sobolevskii, A. 2003, A\&A, 406, 393

Moutarde, F., Alimi, J.-M., Bouchet, F. R., Pellat, R., \& Ramani, A. 1991, ApJ, 382,377

Peebles, P. J. E. 1980, The Large-scale Structure of the Universe (Princeton Univ. Press)

Sahni, V., \& Coles, P. 1995, Phys. Rep., 262, 1

Shandarin, S. F. 1980, Astrofizika 16, 769; 1981, Astrophysics, 16, 439

Serrin, I. 1959, In Encyclopedia of Physics, Vol. III.1, ed. S. Flügge, (Berlin: Springer)

Shirokov, A., \& Bertschinger, E. 2006, ApJS, submitted [arXiv: astro-ph/0505087]

Steinmetz, M. 2003, in Hubble's Science Legacy: Future Optical/Ultraviolet Astronomy from Space, ed. K. R. Sembach, et al., ASP Conf. Proc., 291, 237

Susperregi, M., \& Buchert, T. 1997, A\&A, 323, 295

Tatekawa, T., Suda, M., Maeda, K., Morita, M., \& Anzai, H. 2002, Phys. Rev. D66, 064014

Tatekawa, T. 2004a, Phys. Rev. D, 69, 084020

Tatekawa, T. 2004b, Phys. Rev. D, 70, 064010

Tatekawa, T. 2005a, Phys. Rev. D, 71, 044024

Tatekawa, T. 2005b, Phys. Rev. D, 72, 024005

Vanselow, M. 1995, Diploma Thesis, Ludwig-Maximilians-Universität München (in German)

Zel'dovich, Ya. B. 1970, A\&A, 5, 84

Zel'dovich, Ya. B. 1973, Astrophysics, 6, 164 